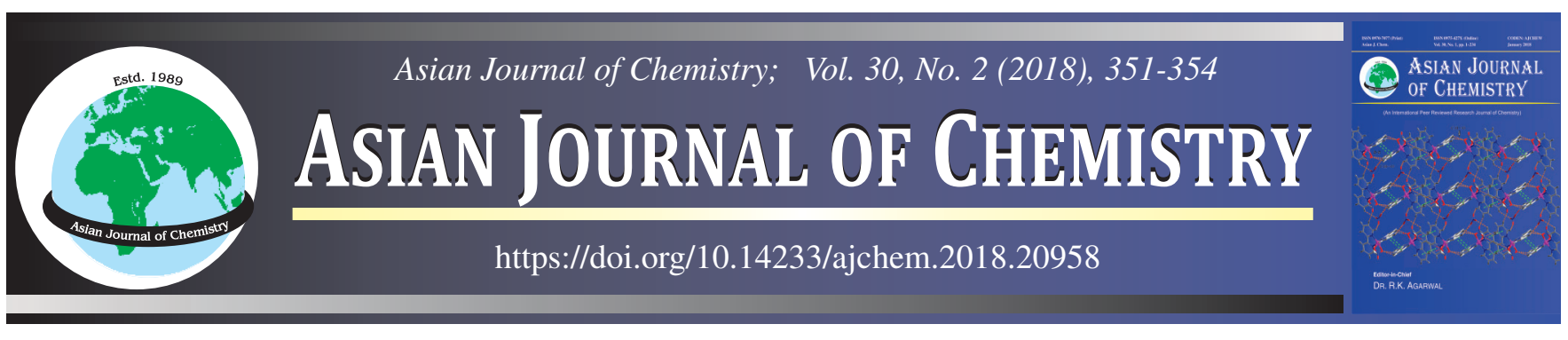

\title{
Light Emitting Diode Based Evanescent Wave Fiber \\ Optic Chemical Sensor for Detection of Thiocyanate
}

\author{
V.K. KulKarni ${ }^{1}$, H.H. Bendigeri ${ }^{2, *}$ and R.M. KulKarni ${ }^{3}$
}

${ }^{1}$ Department of Physics, Angadi Institute of Technology and Management, Belagavi-591 108, India

${ }^{2}$ Department of Physics, K.L.S. Gogte Institute of Technology (Autonomous), Belagavi-590 008, India

${ }^{3}$ Department of Chemistry, K.L.S. Gogte Institute of Technology (Autonomous), Belagavi-590 008, India

*Corresponding author: E-mail: harishbendigeri@git.edu

Received: 7 August 2017;

Accepted: 19 November 2017;

Published online: 31 December 2017;

AJC-18704

The work describes the development of a cost effective and highly sensitive evanescent wave fiber optic chemical sensor for the detection of toxic weak acid dissociable of cyanide i.e. thiocyanate (-SCN) using light emitting diode. High sensitivity is achieved by using unclad portion of multimode sensor grade plastic clad silica fiber as a sensing element. Two photo detectors have been employed to detect output of core and cladding modes. Comparison study gives a description for sensitiveness over the range from 0.02 to $0.1 \mathrm{ppm}$ of thiocyanate concentration.

Keywords: Thiocyanate, Evanescent wave, Chemical sensor, Optical fiber, Cyanide and Light emitting diode.

\section{INTRODUCTION}

A huge expansion in the industrialization and population there is an exponential increase in the waste generation causing the inclusion of number of trace elements and pollutants in to the environment. Precise measurements of these trace elements are vital because of their toxic effects on human life and environment. There are several trace elements including ions of heavy metals, organic compounds and different physical impurities can be present in the water. However these physical impurities can be detected and treated with conventional methods. On the other side, it is difficult to identify trace ions and organic compounds.Hence group of researchers have shown keen interest and have been working on study and development of easy and cost effective detection methods for these pollutants [1,2].

Increasing in the use of cyanide $(\mathrm{CN})$ compounds in mining and related industries, results in many issues related to health of environment and aquatic life. Hence detection of cyanide and cyanide complexes in effluents of mining and related industries is very important. Cyanide ion, consists of carbon and it reacts with trace metals and sulfur like elements to form cyanide complexes. There are two categories of complexes of cyanide (1) WAD- Weak acid dissociable (2) SAD- Strong acid dissociable, these include cyanates and thiocyanate. These two are the major cyanide complexes regularly found in effluents of mining and related industries [3,4].
Thiocyanate $(-\mathrm{SCN})$ is a sulfur-complexed cyanide, it is a linear electronegative polyatomic ion. Though it is a byproduct formed in the process of detoxification of cyanide in the mining industries, on its own it has some application in different industrial processes like manufacturing of thiourea, photofinishing, metal separation and electroplating [2-5]. If the effluents are with high acidic condition the thiocyanate degrades into cyanide ion and sulfur or thiocyanate combines with metals to form metal thiocyanate complexes [6]. Thiocyanate is highly toxic in the weak acid dissociables of cyanide. Its toxicity increases with increase in the concentration of thiocyanate. History of effects of thiocyanate toxicity includes confusion or psychosis, hyferflexia, muscle cramps, seizures, coma, inhibition of variety of enzymes [5,6].

However data related to water treatment shows that many industries have neglected the presence of thiocyanate in water. This laxity of industries increases the health issues. Therefore it is necessary to detect thiocyanate at lower concentration. There are various methods such as analytical [7] spectrophotometric analysis. High performance liquid chromatography with flourimetric method [8] and surface enhanced Raman spectroscopy (SERS) analysis $[9,10]$ have reported on the detection of thiocyanate. But these all are high end, highly versatile, time consuming and off field techniques. This endorsed us to think on easy, cost effective and highly sensitive sensor based on fiber optic technology. 
Fiber optic sensors (FOS): Compared to above mentioned expensive methods, sensing systems based on fiber optic technology have great prospective applications especially in real time or online measurements or sensing applications. Different types of fiber optic sensors have been developed to examine ecological pollutants [11]. Therefore, it is decided to design an ideal and cost effective fiber optic sensors on intensity modulation technique called evanescent wave fiber optic chemical sensor [EWFOCS].

Evanescent wave fiber optic chemical sensor (EWFOCS): In recent years, there has been a significant progress in the field of fiber optic sensors. There are different types of fiber optic sensors for the detection of various chemical species. The mechanism discussed in this work is modulation of optical intensity by exploiting the evanescent wave from unclad portion of the fiber to surrounding media $[12,13]$.

Theory of evanescent wave fiber optic chemical sensor: Exponentially decreasing wave field of light travelling through unclad fiber is called as evanescent wave. This is exploited to surrounding area in designing and development of the EWFOCS. The uncladed fiber acts as an evanescent wave sensor. EWFOCS works on the attenuated total reflection (ATR) i.e. when light travels in the unclad fiber, the amplitude of the totally internally reflected wave decreases due to absorption by surrounding media. Power transmitting in the optical fiber over an unclad portion of length $\mathrm{L}$ is given by,

$$
\mathrm{P}(\mathrm{L})=\mathrm{P}(0) \exp (-\gamma \mathrm{CL})
$$

where $\mathrm{P}(0)$ is initial power in the fiber, $\gamma$ is evanescent wave absorption coefficient; $\mathrm{C}$ is concentration of absorbing medium.

The evanescent wave absorption coefficient can be obtained by Beer-Lambert's law using following relation:

$$
\gamma=\alpha \mathrm{L} \eta
$$

where, $\alpha$ is the absorption coefficient, $\mathrm{L}$ is the interaction length and $\eta$ is the fraction of light in the evanescent field.

The penetration depth (dp) of evanescent wave that enters into cladding from uncladed portion is defined as the distance at which the strength of electric field exponentially decays to $(1 / \mathrm{e})^{\text {th }}$ value and is given by:

$$
\mathrm{dp}=\frac{\mathrm{A}}{2 \pi}
$$

Eqns. 1-3 represents that in EWFOCS sensitivity depends on the dimensions, geometry and other physical properties of the fiber and also on other parameters like incident angle, critical angle, wavelength of the light and absorption coefficient of analytic. As this is one of the sensitive techniques to detect water trace elements, we have selected EWFOCS method to detect thiocyanate $(-\mathrm{SCN})$.

\section{EXPERIMENTAL}

A sensor grade step index multimode Plastic clad Silica (PCS) fiber (F-MBC) procured from NewPort USA has a numerical aperture (NA) of 0.37, diameter of 1000 and 1035 $\mu \mathrm{m}$ for core and cladding respectively. Cladding part of which was taken out physically with the help of sharp razor over a length of $10 \mathrm{~cm}$ which acts as a sensing element.
The sensor cell is made up of glass tube of capacity $25 \mathrm{~mL}$. It is designed in such a way that its upper portion of length around $10 \mathrm{~cm}$ is taken out such that it appears like flat surface in the upper-side of sensor cell. Sensor cell has the provision of one-inlet and one-outlet at upper and lower side respectively. Two holes are at the either ends to pass the optical fiber such that unclad portion of the fiber is exposed at the center of sensor cell.

The chemicals procured are nitric acid (Loba Chemie), ammonium thiocyanate (Loba Chemie) and ferric nitrate (Loba Chemie) are of analytical reagent grade. Standard stock solutions of ammonium thiocyanate and ferric nitrate were prepared using $10 \%$ of acidic medium and diluted using distilled water. Test solutions of different concentrations of thiocyanate from 0.02 to $0.1 \mathrm{ppm}$ were prepared by taking the ammonium thiocyanate solution in $50 \mathrm{~mL}$ measuring flask and adding excess of ferric nitrate solution to get red coloured thiocyanate complex with $\mathrm{Fe}^{3+}$, according to the equation below:

$$
\mathrm{SCN}^{-}(\mathrm{aq})+\mathrm{Fe}^{3+}(\mathrm{aq}) \longrightarrow[\mathrm{FeSCN}]^{2+}
$$

This colour of the complex varies with variation in the thiocyanate concentration. Absorption spectra of different concentrations of test solutions were studied with 50 Bio UV-visible spectrophotometer, procured from Varian. It is clearly visible from Fig. 1 that the absorption peak is at 461 $\mathrm{nm}$. Absorption is studied for 10 to $2 \mathrm{ppm}$ concentration of thiocyanate.

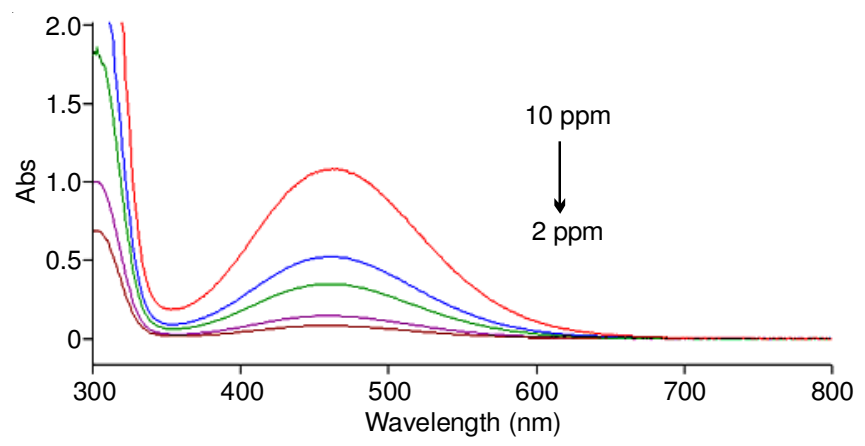

Fig. 1. Absorption spectrum of the test solution consisting various concentration of thiocyanate

To measure the output power of the signal, a power meter model-2936C, with dual channel has been procured from NEWPORT, USA. Other necessary optical components have been purchased from Holmarc, Cochin, India. Green colour LED is coupled to the optical fiber using coupler consisting high quality microscope objective. A sensor grade multimode PCS fiber (F-MBC) with unclad region of $10 \mathrm{~cm}$ is placed at the center of sensor cell. Two photo detectors, model 918DUV-OD and 818-UV/DB both are UV enhanced Si photo detectors have been procured from NEWPORT, USA have been used in this technique to study the sensitiveness. One detector was placed at the end of an optical fiber to record core mode and another detector placed on the open face of the sensor cell to detect the power of evanescent wave coming from unclad portion of the fiber. These two detectors have been used to detect light from core mode and cladding mode respectively and connected to optical power meter as shown in Fig. 2. 


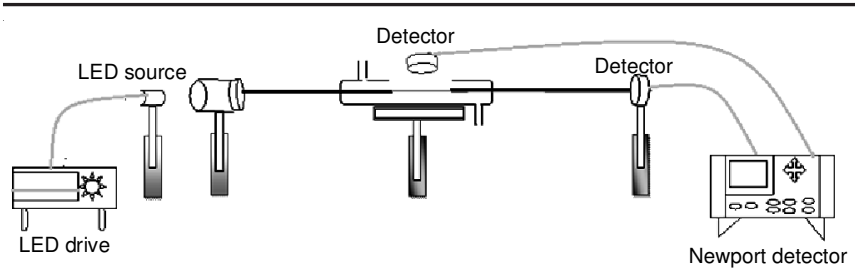

Fig. 2. Experimental set up

Initially output power is recorded for air and distilled water respectively, then recorded for various concentrations from minimum concentration to higher concentration in the order of $0.02 \mathrm{ppm}$ to $0.1 \mathrm{ppm}$. After adding each solution, waited for 2 min to record the readings. After each recording, sensor cell was rinsed with distilled water to prevent the interference of the different concentration of test solutions.

When the test solution is added to sensor cell the unclad portion of PCS fiber is exposed to the surrounding test solution. As the incident wavelength of LED light passing through the fiber almost nearer to the absorption peak of the test solution, a very strong absorption of evanescent field takes place by surrounding media and this absorption increases with increase in concentration of thiocyanate.

\section{RESULTS AND DISCUSSION}

Fig. 3 shows the variation of output power of light signal coming from core component of the fiber with the variation in concentration of thiocyanate. Plot illustrates the linear fit with regression coefficients $\mathrm{R}^{2}=0.987$ and evidently speaks about the sensitiveness of the sensor well below the detection limit of spectrophotometer (Fig. 1). This exposed the interaction of evanescent field with ions of thiocyanate and absorption of evanescent wave field by surrounding media.

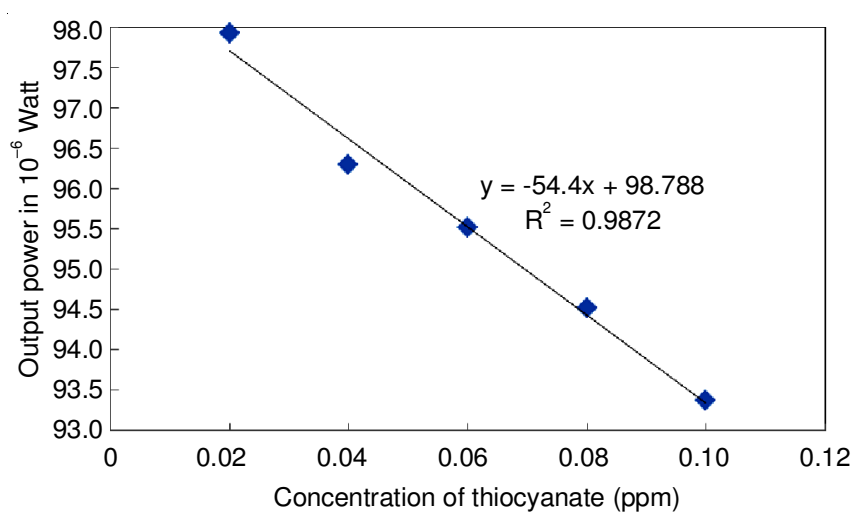

Fig. 3. Variation of guided optical power in the core of an unclad multimode PCS fiber with respect to thiocyanate concentration

Fig. 4 represents the variation in the output power of evanescent wave field with the concentration of thiocyanate. Graph reveals the visualization of evanescent wave and its absorption or decay as the concentration of surrounding media varies. The linear fit with regression coefficient $\left(R^{2}\right)=0.984$ of this graph enlightens that the absorption of evanescent wave depends upon the concentration of thiocyanate, as the concentration increases more and more absorption of evanescent wave or field takes place results in linear decay in the output power of the evanescent wave. As an evident for Figs. 3 and 4 we calculated evanescent absorbance (A) using the below equation.

$$
\mathrm{A}=\frac{\text { Pclad }}{\text { Po }} \frac{\alpha \mathrm{L}}{2.303}
$$

where $\mathrm{A}$ is the evanescent wave absorbance, $\alpha$ is the attenuation coefficient, $\mathrm{L}$ is the length of the fiber, $\mathrm{P}$ (clad) is the power detected by cladding mode detector and $\mathrm{P}$ (o) is the initial power.

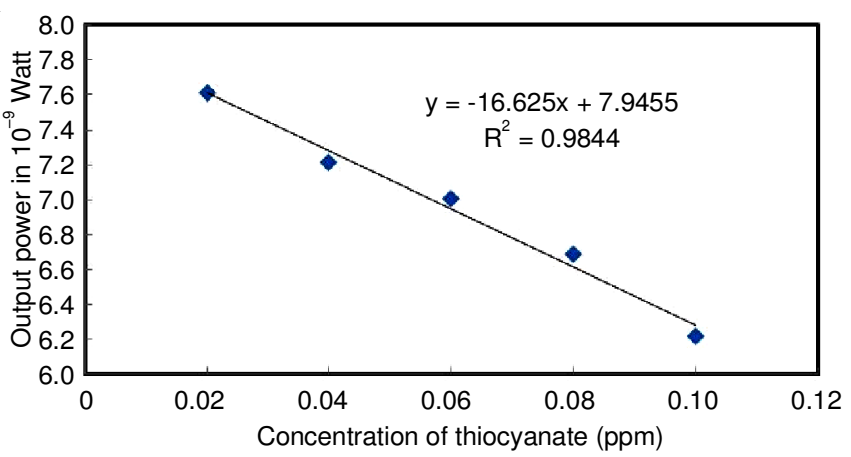

Fig. 4. Variation of guided optical power in the cladding region of an unclad multimode PCS fiber with respect to thiocyanate concentration

Fig. 5 shows the linear variation with the regression coefficient $\left(R^{2}\right)=0.985$ of evanescent wave absorbance against thiocyanate concentration. All these graph shows that device or sensor can respond over the dynamic range from 0.02 to 0.1 ppm and even at very low concentration, linear behaviour of all the graphs obeys Beers-Lamberts law.

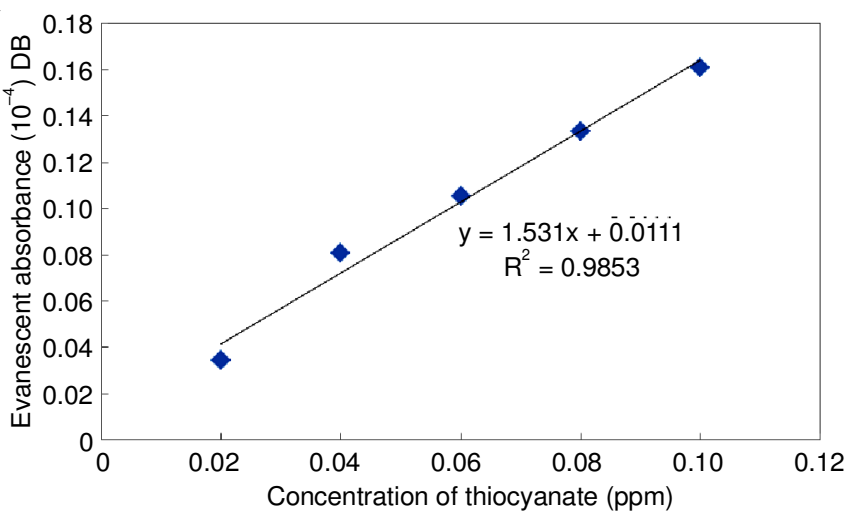

Fig. 5. Variation of evanescent wave absorbance with various concentration of thiocyanate

\section{Conclusion}

We have developed a simple method with EWFOCS technique to detect thiocyanate at lower concentration. In this paper we have shown how evanescent wave field in optical fiber can be successfully exploited for the detection of trace amount of thiocyanate in water. The results obtained using the core mode, cladding mode and spectrophotometer were used for comparative study, which revealed that this EWFOCS technique is highly sensitive and useful for real time or in situ measurement of thiocyanate and can detect low concentration of the order of ppm level. It shows that core and cladding mode detection is very sensitive over spectrometer method. This sensor is of 
intensity modulated type and hence requires only few optical components and also the use of LED source significantly reduces its cost. The stability and reproducibility was studied. This method involves double detection technique which has advantages over single detection method and consequently the accuracy and reliability of the sensor is enhanced in this work. So this technique can be employed to detect thiocyanate in the effluents of mining and related industries for the real time measurements at anywhere in the work field.

\section{ACKNOWLEDGEMENTS}

This work was supported by Visveswaraya Technological University under VTU Research Grants and KLS Gogte Institute of Technology (partial), Belagavi, India.

\section{REFERENCES}

1. K. Bhavsar, R. Prabhu and P. Pollard, J. Phys. Conf. Ser., 450, 012011 (2013);

https://doi.org/10.1088/1742-6596/450/1/012011.

2. A.G. Mignani, A.A. Mencagalia, A. Andrea and L. Ciaccher, Soil and Water Pollution Monitoring, Protection and Remediation, Springer Publication, pp. 3-23 (2006).

3. O. Agrawal, G. Sunita and V.K. Gupta, J. Chin. Chem. Soc. (Taipei), 52 51 (2005); https://doi.org/10.1002/jccs.200500008.
4. V.K. Sharma, Eur. J. Mineral Process. Environ. Prot., 3, 301 (2003).

5. V.K. Sharma, C.R. Burnett, B.D. O' Connor and D. Cabelli, Environ. Sci. Technol., 36, 4182 (2002); https://doi.org/10.1021/es020570u.

6. K. Bosecker and P. Blumenroth, Microbial Treatment of Cyanide and Heavy Metals Containing Waste Water from Gold Mining, $17^{\text {th }}$ International Mining Congress and Exhibition of Turkey - IMCET'2001 (2001).

7. S.-H. Chen, Z.-Y. Yang, H.-L. Wu, H.-S. Kou and S.-J. Lin, J. Anal. Toxicol., 20, 38 (1996);

https://doi.org/10.1093/jat/20.1.38.

8. P. Lundquist, B. Kagedal and L. Nilsson, Eur. J. Clin. Chem. Clin. Biochem., 33, 343 (1995); https://doi.org/10.1515/cclm.1995.33.6.343.

9. E. Saussereau, J.-P. Goullé and C. Lacroix, J. Anal. Toxicol., 31, 383 (2007); https://doi.org/10.1093/jat/31.7.383.

10. Y. Miura and A. Kawaoi, J. Chromatogr. A, 884, 81 (2000); https://doi.org/10.1016/S0021-9673(00)00221-1.

11. S. Chong, A. Aziz and S. Harun, Sensors, 13, 8640 (2013); https://doi.org/10.3390/s130708640.

12. W. Cao and Y. Duan, Sens. Actuators B Chem., 110, 252 (2005); https://doi.org/10.1016/j.snb.2005.02.015.

13. S.T. Lee, P.S. Kumar, K.P. Unnikrishnan, V.P.N. Nampoori, C.P.G. Vallabhan, S. Sugunan and P. Radhakrishnan, Meas. Sci. Technol., 14, 858 (2003); https://doi.org/10.1088/0957-0233/14/6/322. 